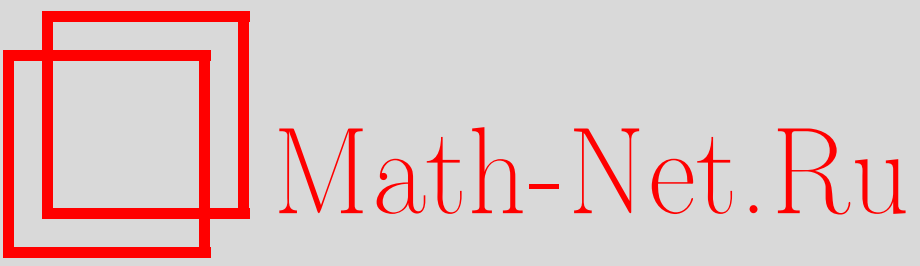

Д. В. Миллионщиков, Деформации градуированных алгебр Ли и симплектические структуры, УМН, 2003, том 58, выпуск 6, 157-158

DOI: https://doi.org/10.4213/rm685

Использование Общероссийского математического портала Math-Net.Ru подразумевает, что вы прочитали и согласны с пользовательским соглашением

http://www.mathnet.ru/rus/agreement

Параметры загрузки:

IP: 54.198 .55 .26

26 апреля 2023 г., 11:50:16 


\title{
ДЕФОРМАЦИИ ГРАДУИРОВАННЫХ АЛГЕБР ЛИ И СИМПЛЕКТИЧЕСКИЕ СТРУКТУРЫ
}

\author{
Д. В. Миллионшиков
}

Симплектические нильмногообразия являются важньм классом примеров симплектических многообразий, не допускающих кэлеровой структуры. Вопрос о существовании симплектической формы на нильмногообразии $G / \Gamma$ решается в терминах алгебры Ли $\mathfrak{g}$ нильпотентной группы Ли $G$. В [1] и [2] было рассмотрено интересное семейство симплектических градуированных нильпотентных алгебр Ли $\mathscr{V}_{n}, n=2 k$ (и соответствующих нильмногообразий), заданных базисом $e_{1}, e_{2}, \ldots, e_{n}$ и коммутационными соотношениями

$$
\left[e_{i}, e_{j}\right]=\left\{\begin{array}{ll}
(j-i) e_{i+j}, & i+j \leqslant n, \\
0, & i+j>n,
\end{array} \quad \omega_{2 k+1}^{\mathcal{V}}=\frac{1}{2} \sum_{i+j=2 k+1}(j-i) e^{i} \wedge e^{j}\right.
$$

где замкнутая 2-форма $\omega_{2 k+1}^{\mathscr{V}}$ определяет симплектическую структуру, а $e^{1}, \ldots, e^{2 k}$ обозначают двойственный к $e_{1}, \ldots, e_{2 k}$ базис 1-форм.

Алгебры $\mathscr{V}_{n}$ являются примером филиформных алгебр Ли - нильпотентных алгебр Ли $\mathfrak{g}$ с максимальной для их размерности $\operatorname{dim} \mathfrak{g}$ длиной $s=\operatorname{dim} \mathfrak{g}-1$ нижнего центрального ряда. Идеалш $\left\{C^{i} \mathfrak{g}=\left[\mathfrak{g}, C^{i-1} \mathfrak{g}\right]\right\}$ нижнего центрального ряда нилппотентной алгебры Ли $\mathfrak{g}$ определяют ее конечную убывающую фильтрацию $C$. Для фильтрованной таким образом филиформной алгебры $\mathfrak{g}$ размерности однородных компонент ее присоединенной градуированной алгебры Ли $\operatorname{gr}_{C} \mathfrak{g}=\bigoplus_{i=1}^{n-1}\left(\operatorname{gr}_{C} \mathfrak{g}\right)_{i}\left(\left(\operatorname{gr}_{C} \mathfrak{g}\right)_{i}=C^{i} \mathfrak{g} / C^{i+1} \mathfrak{g}\right)$ равны: $\operatorname{dim}\left(\operatorname{gr}_{C} \mathfrak{g}\right)_{1}=2, \operatorname{dim}\left(\operatorname{gr}_{C} \mathfrak{g}\right)_{2}=$ $\cdots=\operatorname{dim}\left(\operatorname{gr}_{C} \mathfrak{g}\right)_{n-1}=1$. Простейшим примером филиформной алгебры является алгебра $\mathfrak{m}_{0}(n)$, заданная базисом $e_{1}, e_{2}, \ldots, e_{n}$ и коммутационньми соотношениями $\left[e_{1}, e_{i}\right]=e_{i+1}, i=$ $2, \ldots, n-1$ (соотношения вида $\left[e_{i}, e_{j}\right]=0$ мы будем далее опускать). Алгебра $\mathfrak{m}_{0}(2 k)$ обладает симплектической структурой $\omega_{2 k+1}^{\mathfrak{m}_{0}}=\frac{1}{2} \sum_{i+j=2 k+1}(-1)^{i} e^{i} \wedge e^{j}$.

УтВеРЖДЕнИЕ 1. Пусть $\mathfrak{g}$ - симплектическая филиформная алгебра Ли. Тогда $\operatorname{gr}_{C} \mathfrak{g} \cong \mathfrak{m}_{0}(2 k)$ u, значит, существует базис $e_{1}, \ldots, e_{2 k}$ в $\mathfrak{g}$ (так называемый адаптированный базис, введенный в [3]) такой, что

$$
\left[e_{i}, e_{j}\right]=\sum_{l=0}^{2 k-i-j} c_{i j}^{l} e_{i+j+l}, \quad i+j \leqslant 2 k .
$$

Набор идеалов $L^{k} \mathfrak{g}=\left\langle e_{k}, \ldots, e_{n}\right\rangle, k=1, \ldots, n$, определяет фильтрацию $L$ алгебры $\mathfrak{g}$, причем присоединенная градуированная алгебра Ли $\operatorname{gr}_{L} \mathfrak{g}=\bigoplus_{i=1}^{n}\left(\operatorname{gr}_{L} \mathfrak{g}\right)_{i}$ является симплектической $u \operatorname{dim}\left(\operatorname{gr}_{L} \mathfrak{g}\right)_{i}=1, i=1, \ldots, n$.

Алгебра $\operatorname{gr}_{L} \mathfrak{g}$ может быть задана базисом и коммутационньми соотношениями $\left[e_{i}, e_{j}\right]=$ $c_{i j}^{0} e_{i+j}, i+j \leqslant 2 k$. Коцепные комплексы $\left(C^{*}\left(\operatorname{gr}_{L} \mathfrak{g}\right), d\right),\left(C^{*}\left(\operatorname{gr}_{L} \mathfrak{g}, \operatorname{gr}_{L} \mathfrak{g}\right), d\right)$ и соответствующие когомологии допускают вторую градуировку (вес) $C^{*}\left(\operatorname{gr}_{L} \mathfrak{g}\right)=\bigoplus_{p} C_{(p)}^{*}\left(\operatorname{gr}_{L} \mathfrak{g}\right)$, $C^{*}\left(\operatorname{gr}_{L} \mathfrak{g}, \operatorname{gr}_{L} \mathfrak{g}\right)=\bigoplus_{p} C_{(p)}^{*}\left(\operatorname{gr}_{L} \mathfrak{g}, \operatorname{gr}_{L} \mathfrak{g}\right), p\left(e^{i_{1}} \wedge e^{i_{2}} \wedge \cdots \wedge e^{i_{q}}\right)=i_{1}+i_{2}+\cdots+i_{q}$, $p\left(e_{n} \otimes e^{i_{1}} \wedge e^{i_{2}} \wedge \cdots \wedge e^{i_{q}}\right)=n-\left(i_{1}+i_{2}+\cdots+i_{q}\right)$.

УТВЕРЖДЕНИЕ 2. Форма $\omega$ является симплектической для $\operatorname{gr}_{L} \mathfrak{g}$, если и только если $\omega=\omega_{2 k+1}+\omega^{\prime}$, где $\omega_{2 k+1}-$ однородная симплектическая форма веса $2 k+1$, a $\omega^{\prime}-$ произвольный коцикл с однородными слагаемыми с весами $<2 k+1$.

Для доказательства достаточно заметить, что $H_{(p)}^{2}\left(\operatorname{gr}_{L} \mathfrak{g}\right)=0$ при $p>2 k+1$, а $p\left(\omega^{k}\right)=$ $p\left(e^{1} \wedge e^{2} \wedge \cdots \wedge e^{2 k}\right)=k(2 k+1)$. Градуированные филиформные алгебры вида $\operatorname{gr}_{L} \mathfrak{g}$ классифицированы в [4], элементарные когомологические вычисления показывают, что в размерностях

Работа выполнена при поддержке Российского фонда фундаментальных исследований (грант № 02-01-00659) и гранта PAI-Pоссия N 04495UL. 
$2 k \geqslant 12$ есть только две (с точностью до изоморфизма) симплектические градуированные филиформные алгебры: это $\mathfrak{m}_{0}(2 k)$ и $\mathscr{V}_{2 k}$.

В данной заметке мы рассматриваем алгебры $\mathfrak{g}$ такие, что $\operatorname{gr}_{C} \mathfrak{g} \cong \mathfrak{m}_{0}(n), \operatorname{gr}_{L} \mathfrak{g} \cong \mathscr{V}_{n}$. Для каждой из них можно выбрать базис такой, что

$$
\left[e_{i}, e_{j}\right]=(j-i) e_{i+j}+\sum_{l=1}^{n-i-j} c_{i j}^{l} e_{i+j+l} .
$$

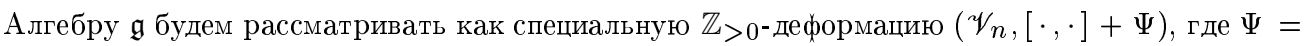
$\Psi_{1}+\Psi_{2}+\cdots+\Psi_{n-3}, \Psi_{l} \in C_{(l)}^{2}\left(\mathscr{V}_{n}, \mathscr{V}_{n}\right)$. Уравнение деформации $d \Psi+\frac{1}{2}[\Psi, \Psi]=0$ можно записать как систему уравнений на однородные компоненты [5]:

$$
d \Psi_{1}=0, d \Psi_{2}+\frac{1}{2}\left[\Psi_{1}, \Psi_{1}\right]=0, \ldots, d \Psi_{n-6}+\frac{1}{2} \sum_{i+j=n-6}\left[\Psi_{i}, \Psi_{j}\right]=0 .
$$

УТвеРЖДЕНИЕ 3. Полупрямое произведение $G_{n}=\mathbb{K}^{*} \ltimes U T_{n}$ подгруппь $\mathbb{K}^{*}$ диагональных матрич $\left\{\alpha^{i} \delta_{j}^{i}\right\}$ и подгруппь $U T_{n}$ нижнетреугольных матрич с единицами на главной диагонали действ ует на аффинном многообразии $V_{n} \mathbb{Z}_{>0}$-деформаций $\mathscr{V}_{n}$ заменами канонического базиса: $\varphi\left(e_{i}\right)=\alpha^{i} e_{i}+\sum_{j>i} a_{i j} e_{j}$. Две $\mathbb{Z}_{>0}$-деформации $\left(\mathscr{V}_{n},[\cdot, \cdot]+\Psi\right) u$ $\left(\mathscr{V}_{n},[\cdot, \cdot]+\widetilde{\Psi}\right)$ задают изоморфнье алгебры Ли тогда и только тогда, когда они лежат в одной орбите $O_{\Psi}$ әтого $G_{n}$-действия.

Рассмотрим убьвающую фильтрацию $\widetilde{L}$ комплекса $\left(C^{*}\left(\mathscr{V}_{n}, \mathscr{V}_{n}\right), d\right)$, где подпространство $\widetilde{L}^{k} C^{*}\left(\mathscr{V}_{n}, \mathscr{V}_{n}\right)$ порождено формами $c$ такими, что $\operatorname{Im} c \subset L^{k} \mathscr{V}_{n}$. Фильтрация $\widetilde{L}$ определяет спектральную последовательность $E_{r}^{p, q}$, сходящуюся к $H^{*}\left(\mathscr{V}_{n}, \mathscr{V}_{n}\right)$.

ЛЕмма 1. Пусть $n \geqslant 16$, тогда $\operatorname{dim} H^{2}\left(\mathscr{V}_{n}, \mathscr{V}_{n}\right)=10$, в частности, подпространство $\bigoplus_{i>0} H_{(i)}^{2}\left(\mathscr{V}_{n}, \mathscr{V}_{n}\right)$ порождается кочиклами $\psi_{n, i}$, соответствующими әлементам $\left[\psi_{n, i}\right]=e_{n+7-i} \otimes\left[e^{2} \wedge e^{5}-3 e^{3} \wedge e^{4}\right], i=11, \ldots, 7$, из $E_{1}=\mathscr{V}_{n} \otimes H^{*}\left(\mathscr{V}_{n}\right)$.

Часть коциклов $H^{2}\left(\mathscr{V}_{n}, \mathscr{V}_{n}\right)$ с отрицательньми весами найдена в [6].

УТВЕРЖДЕнИЕ 4 . При $n \geqslant 16$ многообразие $V_{n} \mathbb{Z}_{>0}$-деформаций содержит 5-мерную плоскость $\Gamma_{n} \cong \mathbb{K}^{5}=\left\{x_{1} \psi_{n, 11}+\cdots+x_{5} \psi_{n, 7}\right\}$. Произвольная орбита $O_{\Psi}^{\prime}$ действия $U T_{n}$ на $V_{n}$ пересекает $\Gamma_{n}$ в единственной точке.

Теорема 1. При $n \geqslant 16$ существует взаимно однозначное соответствие между множеством $\mathscr{M}_{n}$ классов изоморфных $\mathbb{Z}_{>0}$-деформаций $\mathscr{V}_{n}$ и пространством орбит $O\left(G_{n} / U T_{n}, \Gamma_{n}\right)$, где $G_{n} / U T_{n}=\mathbb{K}^{*}$ действует на $\Gamma_{n}=\mathbb{K}^{5}$ следующим образом:

$$
\widetilde{\rho}_{n}(\alpha)\left(x_{1}, x_{2}, \ldots, x_{5}\right)=\left(\alpha^{n-11} x_{1}, \alpha^{n-10} x_{2}, \ldots, \alpha^{n-7} x_{5}\right), \quad \alpha \in \mathbb{K}^{*} .
$$

В четномерном случае подпространство симплектических алгебр выделяется уравнением $x_{1}=0$.

\section{СПИСОК ЛИТЕРАТУРЫ}

[1] И. К. Бабенко, И. А. Тайманов // УМН. 1998. Т. 53. № 5. С. 225-226. [2] В. М. Бухштабер // УМН. 1999. Т. 54. № 4. C. 161-162. [3] M. Vergne // Bull. Soc. Math. France. 1970. V. 98. Р. 81-116. [4] Д. В. Миллионщиков // УМН. 2002. Т. 57. № 2. С. 197-198. [5] А. Nijenhuis, R. W. Richardson, Jr. // J. Math. Mech. 1967. V. 17. № 1. Р. 89-105. [6] А. Фиаловски // УМН. 1983. Т. 38. № 1. С. 201-202. 